Final progress report for

\title{
THERMOPHYSICAL PROPERTIES OF FLUIDS AND FLUID MIXTURES
}

PI: George Stell

For the funding period $12 / 01 / 06$ to $7 / 31 / 2008$

Grant DE-FG02-88ER13850

Recipient organization:

The Research Foundation of State University of New York

SUNY at Stony Brook

Stony Brook, NY 1179-3362

No unexpended funds were left at the end of the final budget period, which ended $7 / 26 / 07$. The aims of the funded research were unchanged from those of the original application. 


\section{Final Report}

At the direction of our Department of Energy - Basic Energy science Program Director, our DOE supported research was closed out as of 12/01/06. Our work had been the theoretical statistical-mechanical based study of the thermophysical properties of fluids and fluid mixtures.

We continued that part of our research involving self-supporting collaborators at other sites, namely Alina Ciach in Warsaw, Poland, Johan Hoye in Trondheim, Norway, and Dor Ben-Amotz at Purdue University. We used the $\$ 20,000$ close-out money we were granted to support our post-doc, Kippi Dyer, in a transitional period during which we arranged alternative funding for him. On 7/26/07 we were granted a no-cost extension of the grant's termination date to $2 / 29 / 08$. This is our final report prepared as of that date. 